\title{
Fußdeformitäten im Kindesalter - Wann behandeln? Wie behandeln? - Teil 2
}

\author{
L. Döderlein, C. Multerer \\ Orthopädische Kinderklinik am Behandlungszentrum Aschau GmbH
}

Die Operation darf nicht als isolierte Therapiemaßnahme oder als „Ultima Ratio“ angesehen werden, sondern sie bildet eine zusätzliche Möglichkeit im Behandlungsspektrum, wenn die bisher angewendeten Techniken nur unzureichend oder nicht mehr greifen. Operationen sind fast immer mit krankengymnastischer und orthetischer Nachbehandlung zu kombinieren und damit auch mehrdimensional ausgerichtet.

\section{Operative Therapiemethoden}

\section{Allgemeine Grundlagen}

Es gibt verschiedene Operationsarten, die am Kinderfuß zum Einsatz kommen. Sie lassen sich wie folgt unterteilen:

- Operationen an Sehnen und Muskeln (an der MuskelSehnen-Einheit),

- Operationen an Bändern und Gelenkkapseln,

- Operationen an Gelenken,

- Operationen an Knochen,

- kombinierte Eingriffe.

Das Wirkungsspektrum dieser Maßnahmen ist stark unterschiedlich, was ihre Kombination sinnvoll machen kann und bei der Indikationsstellung ausreichend berücksichtigt werden muss.

Operationen an Sehnen und Muskeln bestehen in Sehnen- und Muskelverlängerungen, in Sehnenraffungen, Sehnenfesselungen und Sehnentranspositionen:

- Sehnen- und Muskelverlängerungen haben das Ziel einer Verbesserung der Exkursion der vom Muskel überbrückten Gelenke.

- Sehnenraffungen dienen der Verkürzung zu lang gewachsener Sehnen-Muskel-Einheiten und haben damit das Ziel, durch die Straffung der Antagonisten eine bessere Agonisten-Antagonisten-Balance wiederherzustellen.

- Sehnenfesselungen (Tenodesen) dienen einer externen Stabilisierung von muskulär nicht ausreichend geführten Gelenken.

- Sehnentranspositionen sind zur dynamischen Balancierung von muskulären Ungleichgewichten angezeigt. Die zugehörigen Deformitäten müssen vor jeder Transposition passiv korrigierbar und die Gelenke ausreichend stabil sein. Ein versetzter Muskel kann keine strukturelle Deformität korrigieren, was leider oft nicht beachtet wird und die Methode in Misskredit bringt.

Gelenkeingriffe lassen sich in Gelenklösungen (Kapsulotomien) und Gelenkraffungen (Kapsulodesen) einteilen:

- Eine Kapsulotomie ist bei struktureller Gelenkkontraktur indiziert,

- eine Kapselraffung (Kapsulodese) bei einer Gelenkinstabilität mit Elongation der zugehörigen Gelenkkapsel.

Beim angeborenen Klumpfuß oder beim Talus verticalis kommen Kapsulotomien des oberen und des unteren Sprunggelenks zum Einsatz, beim Knick-Platt-Fuß dagegen Kapsulodesen des Pfannenbands. 
Gelenkeingriffe werden in die Techniken der Anschlagsperre (Arthrorise) und der Gelenkstabilisierung (Arthrodese) unterteilt:

- Die Anschlagsperre verfolgt das Ziel, eine pathologisch gesteigerte Überbeweglichkeit eines Gelenks durch einen künstlich geschaffenen Anschlag auf normale Bewegungsumfänge zu reduzieren.

- Arthrodesen werden beim Kinderfuß nur selten eingesetzt. Sie bewirken eine Korrektur einer störenden Gelenkbewegung oder pathologischen Gelenkstellung durch die knöcherne Versteifung in einer funktionellen Mittelstellung. Das Anwendungsgebiet dieses eingreifenden Verfahrens liegt bei neurologisch bedingten Fußdeformitäten ohne Muskelgleichgewicht oder schweren und steifen Rezidivdeformitäten, die eine ausreichende funktionelle Beweglichkeit nicht mehr erwarten lassen (z. B. bei Rezidivklumpfüßen oder Ballenhohlfüßen). Es sollte immer daran gedacht werden, dass eine ausreichende Gelenkbeweglichkeit auch ein entsprechendes Muskelgleichgewicht erfordert, das das betreffende Gelenk um die Mittelstellung bewegt und sichert.
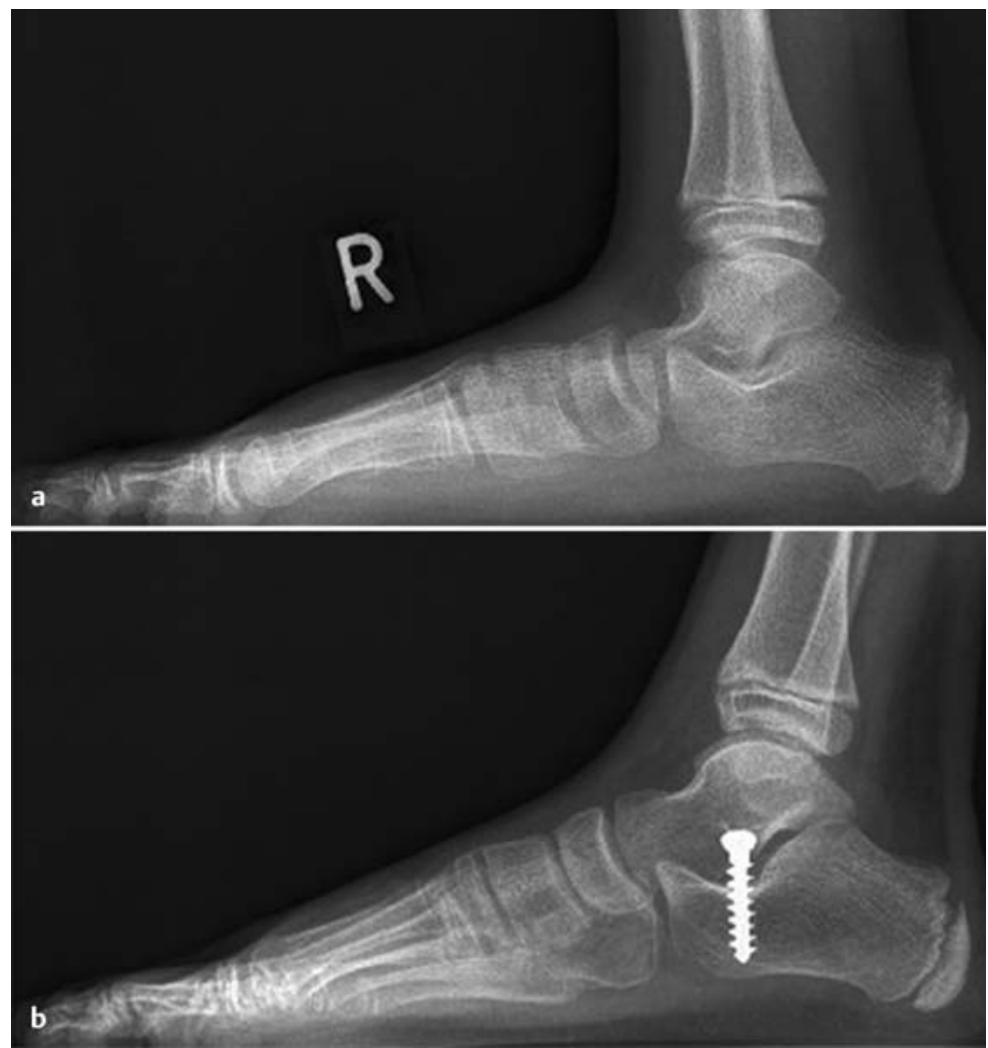

Abb. 1 - Arthrorise eines lockeren Knick-Platt-Fußes mit Spongiosaschraube, präoperativ (a) und postoperativ (b).

\section{Spezielle Verfahren beim Klumpfuß}

Die Behandlung nach Ponseti umfasst nach abgeschlossener Gipsredression neben der nahezu stets durchzuführenden Tenotomie der Achillessehne und der Plantarissehne besonders bei einer Rezidivneigung auch die Lateralverlagerung des Ansatzes der Tibialis-anteriorSehne auf das Os cuneiforme laterale.

Die sog. peritalare Arthrolyse stellt die klassische Klumpfußoperation dar und hat das Ziel, die strukturelle Medialrotation (Verwringung) der subtalaren Fußplatte wieder zu normalisieren. Dazu müssen die Gelenke des Rückfußes gelöst und in korrekter Stellung temporär durch Kirschner-Drähte fixiert und die Achillessehne und die Tibialis-posterior-Sehne dosiert verlängert werden. Der Fuß muss im Oberschenkelgips 6 Wochen lang immobilisiert werden.

\section{Spezielle Verfahren beim Knick-Platt-Fuß}

Die Methode der Gelenksperre (Arthrorise) hat sich als wenig invasives Verfahren für flexible und symptomatische Knickfüße durchgesetzt. Die Sperre gegen eine übermäßige Eversion des Rückfußes wird entweder durch eine 6,5 mm starke Spongiosaschraube (Abb. 1) am Vorderrand des hinteren unteren Sprunggelenks oder durch einen in den Sinus tarsi eingebrachten expandierbaren Dübel (Spacer) bewerkstelligt. Eventuell vorhandene Begleitdeformitäten im Vorfußbereich (z.B. Supination) oder eine Wadenmuskelverkürzung müssen gesondert angegangen werden.

Strukturelle Knick-Platt-Füße müssen in allen ihren Komponenten korrigiert werden. Dies bedeutet, dass die übermäßige peritalare Entwringung wieder auf ein normales Maß vermindert werden muss. Dazu dienen für die transversale Komponente der Deformität die Angleichung der medialen und der lateralen Fußsäule über die Kalkaneusverlängerung nach Evans (Abb.2). Eine verstärkte Vorfußsupination muss durch eine pronierende Vorfußosteotomie (z.B. am Os cuneiforme mediale) korrigiert werden, eine verstärkte Eversion des Kalkaneus durch eine medialisierende Verschiebeosteotomie.

Beim angeborenen Plattfuß, der durch die „reversed Ponseti-Technik“ bereits konservativ weitgehend korrigiert werden konnte, schließt sich ein kleiner operativer Eingriff an. Konnte das Talonavikulargelenk konservativ komplett reponiert werden, wird es mit Kirschner-Drähten perkutan fixiert und danach eine Achillotenotomie durchgeführt. Konnte das Talonavikulargelenk konservativ nicht reponiert werden, erfolgt dies offen über 
einen kleinen medialen Zugang. Bei starker Verkürzung der Extensoren- und Peronäalsehnen müssen diese zusätzlich verlängert werden (Dobbs et al. 2007).

Beim kontrakten Talus verticalis oder unzureichender Korrektur durch das vorbeschriebene Manöver muss die gesamte nach dorsolateral luxierte subtalare Fußplatte gelöst und wieder unter den Talus gestellt werden. Der Schlüssel der erfolgreichen Operation liegt in der korrekten Reposition und Retention des Talonavikular- und des Kalkaneokuboidgelenks bei sparsamer Achillessehnen- und Tibialisanterior-Verlängerung.

- Die Wirkprinzipien der verschiedenen Operationstechniken sollten genau bekannt sein, um die für die jeweilige Deformität am besten geeignete Methode auszuwählen. Der Kinderorthopäde muss mehrere Techniken parat haben, um die breite Palette verschiedener Fußdeformitäten behandeln zu können.

\section{Spezielle Punkte zur OP-Aufklärung}

Die Operationsaufklärung muss neben den allgemein üblichen Risiken wie das Narkoserisiko, Wundheilungsprobleme, Überkorrektur und Rezidiv immer auch deformitätsspezifische Punkte beinhalten.

Beim angeborenen Klumpfuß zählt die Überdosierung der Achillessehnenverlängerung bzw. Tenotomie mit nachfolgender Wadenmuskelinsuffizienz zu den wesentlichsten Punkten.

Eine anfangs durch Ponseti-Therapie erfolgreich korrigierte Fehlstellung kann sich wegen der fortbestehenden Pathomechanik der fibrösen Geweberetraktion am Fußinnenrand allmählich wieder in eine Klumpfußdeformität entwickeln. Deshalb müssen die Eltern darüber aufgeklärt werden, dass die Ponseti-Methode gewisse deformitäts- und altersbedingte Grenzen hat und dass ggf. bei einem Rezidiv eine offene Klumpfußoperation durch peritalare Arthrolyse (Abb.3) notwendig werden kann. Dieses Verfahren hat für sich aber weitere Komplikationsmöglichkeiten wie z.B. eine Überkorrektur oder ein erneutes Rezidiv (sog. „rebellischer Klumpfuß“).

Bei der operativen Behandlung des Knick-Platt-Fußes ist zwischen dem lockeren instabilen und dem rigiden steifen Plattfuß (angeborener Schaukelfuß, Plattfuß bei Koalitionen) zu trennen.

Die instabile lockere Deformität trägt insbesondere dann, wenn sie von einer allgemeinen Kollageninsuffizienz (ligamentäre Hyperlaxizität) begleitet wird, stets das Risiko

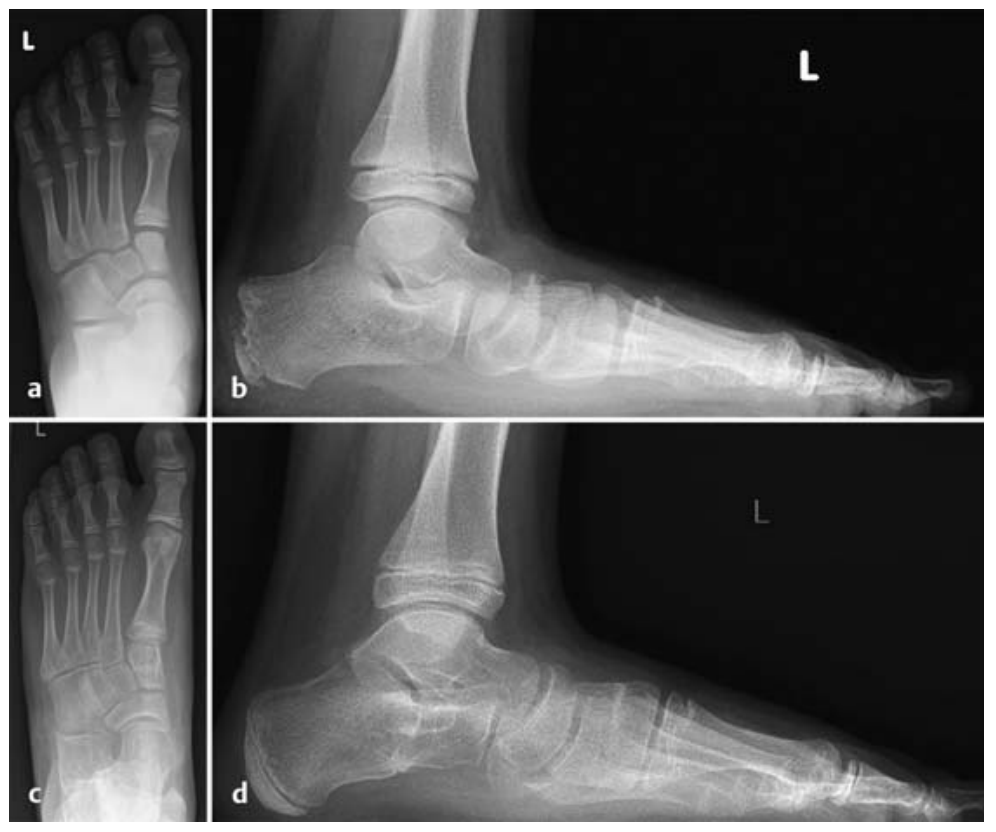

Abb. 2 - Kalkaneusverlängerung nach Evans, präoperativ (a, b) und postoperativ (c, d).

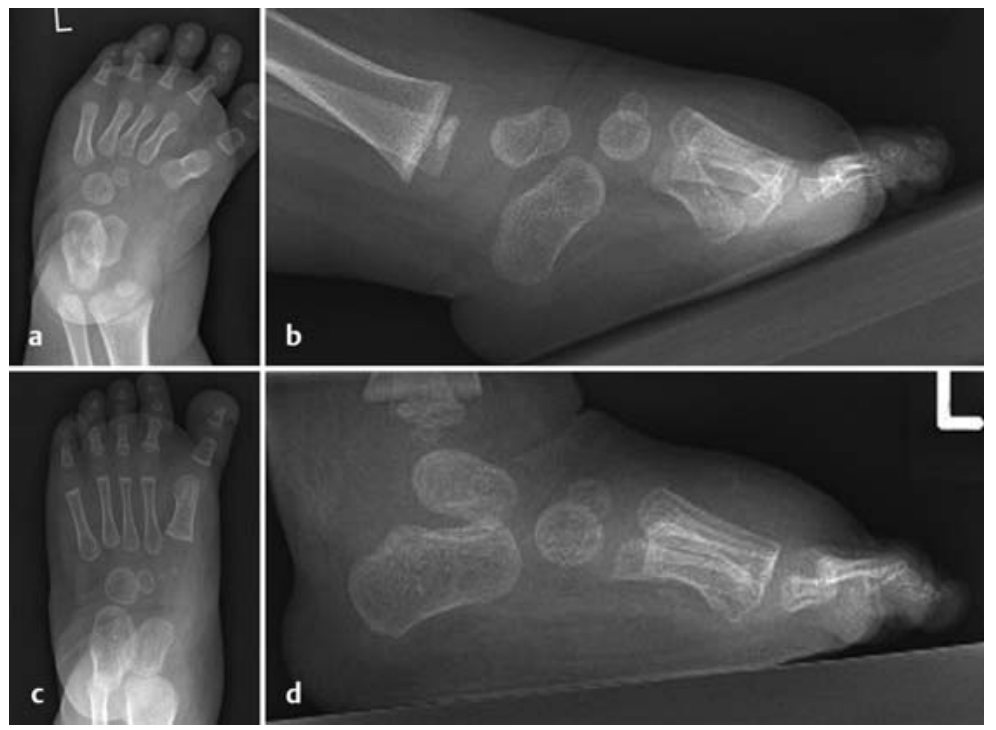

Abb. 3 - Peritalare Arthrolyse bei rigider Klumpfußdeformität präoperativ (a, b) und postoperativ (c, d).

eines Knick-Platt-Fuß-Rezidivs in sich, es sei denn, man hat die Rückfußgelenke in einer Mittelstellung versteift, was aber beim neuromotorisch gesunden Kind nur selten indiziert ist.

Der steife Schaukelfuß trägt ebenfalls das Risiko einer rezidivierenden Deformität in sich, insbesondere dann, wenn er primär nicht vollständig korrigiert werden konnte. Da bei dieser Deformität fast immer auch eine Verlängerung der Achillessehne vorgenommen wird und die Fußheber verkürzt sind, muss auch über das Risiko der Überkorrektur in den Hackenfuß gesprochen werden. 
Ausgedehnte Gelenklösungen im Rückfußbereich beinhalten außerdem das Risiko der dauerhaften Bewegungseinschränkung (durch eine fibröse Gelenksteife = Ankylose) und der vaskulären Talusschädigung (Talusnekrose).

\section{Fallstricke, Tipps und Tricks sowie Fehler und Gefahren bei der Therapie}

Alle ausgeprägten Fußdeformitäten im Kindesalter sind mit gewissen Fallstricken assoziiert, von denen man die wichtigsten kennen sollte.

\section{Diagnostik}

Zuerst muss die Diagnose der Fußdeformität, d.h. ob diese primärer oder sekundärer Natur ist, ausreichend gesichert sein. Dies betrifft nicht nur die Beurteilung der Fußform (Klumpfuß, Knick-Senk-Fuß usw.), sondern immer auch die Abklärung möglicher zusätzlicher pathologischer Komponenten. In diesem Zusammenhang sind vor allem neuromuskuläre Störungen (progredient oder stationär) der unterschiedlichsten Arten zu nennen.

Bei allen einseitigen Knick-Platt-Fuß-Deformitäten ist eine genaue Ursachenanalyse zu fordern. Insbesondere bei subtalarer Bewegungseinschränkung sollte an Koalitionen oder entzündliche/tumoröse Prozesse gedacht werden.
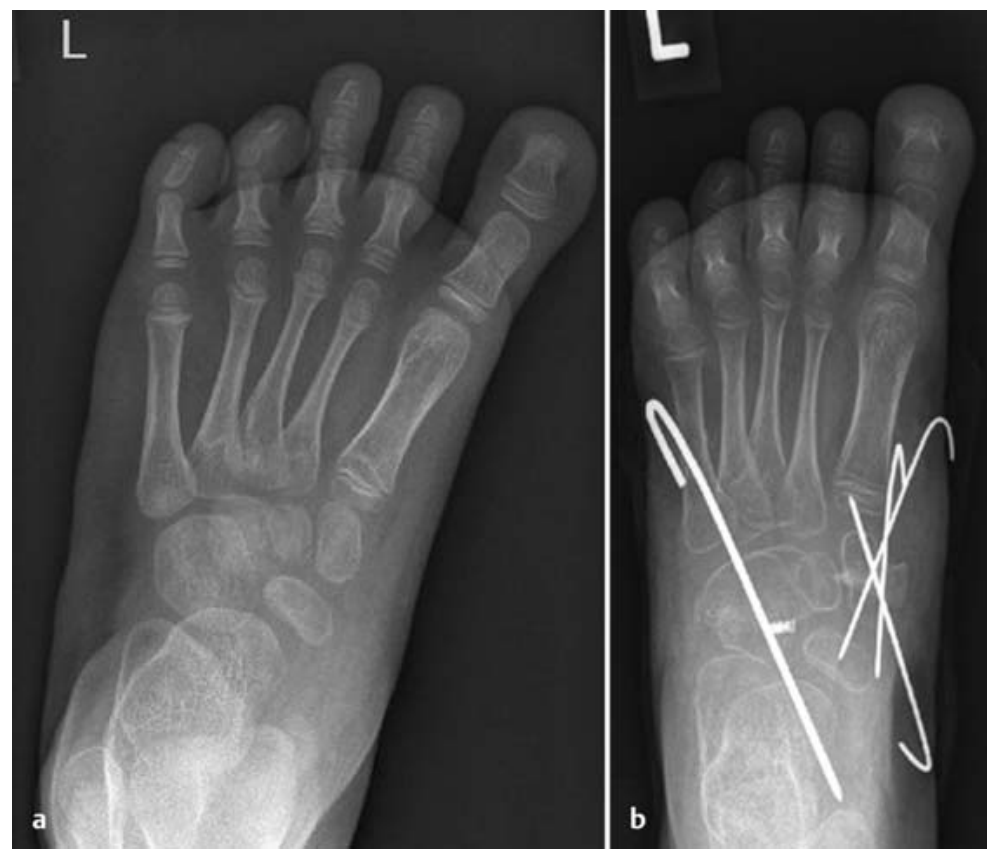

Abb. 4 - Verlängerung des Fußinnenrands und Verkürzung des Fußaußenrands (Operation n. McHale), präoperativ (a) und postoperativ (b).

\section{Therapie}

Die Ponseti-Therapie des idiopathischen (primären) Klumpfußes ist heute ein Standardverfahren in der Kinderorthopädie. Trotzdem ist man überrascht, wie unterschiedlich diese Methode angewendet wird, sei es hinsichtlich der Gipstechnik, der Indikation zur Achillotenotomie oder der Nachbehandlung. Eine fehlerhafte Gipstechnik kann einen Säuglingsklumpfuß problemlos zum ungünstigen Schaukelfuß verformen. Eine unvollständige Achillotenotomie provoziert immer ein Rezidiv, weshalb wir diesen Eingriff grundsätzlich in Kurznarkose und offen durchführen. Ebenso wird die fehlerhafte Orthesenbehandlung ein Wiederauftreten des Klumpfußes begünstigen.

Die Ponseti-Methode eignet sich insbesondere bei kurzen speckigen und rigiden Säuglingsfüßen nur bedingt und mit gewissen Modifikationen. Wenn die Korrektur der Vorfußkavus- bzw. Pronationskomponente nicht ausreichend möglich ist, sollte man eine offene Operation (Plantaraponeurosenablösung) wählen.

Die peritalare Arthrolyse als mediale, laterale und plantare Operationsmethode ist wegen der Gefahr einer Überdosierung heute weitgehend hinter der PonsetiMethode zurückgetreten, dennoch kann man mit dieser Technik bei sorgfältiger Planung und Durchführung auch schwere Klumpfußdeformitäten dauerhaft gut korrigieren. Eine intraoperativ absolut exakte Stabilisierung der erreichten Gelenkstellung durch Kirschner-Drähte unterstützt die adäquate Heilung und erhält die Korrekturstellung.

Sollte nach Klumpfußtherapie im weiteren Verlauf eine Vorfußadduktion und -supination bei neutralem Rückfuß auftreten, wäre eine Operation n. McHale (aufklappende Cuneiforme-I- und zuklappende Kuboidosteotomie) in Kombination mit einem M.-tibialis-anterior-Transfer in Fußrückenmitte indiziert (Abb.4).

Beim sekundären Klumpfuß muss stets neben der Formkorrektur auch eine Muskelbalancierung angestrebt werden, um die Rezidivgefahr zu mindern. Dies betrifft sowohl spastische als auch schlaffe Deformitäten. Instabile oder rigide deformierte Gelenke, für die keine muskuläre Balance geschaffen werden kann, sollten ggf. auch frühzeitig ohne Nachteile für den Patienten stabilisiert werden. Es macht keinen Sinn, bei entsprechend schweren Instabilitäten oder Deformitäten mit knöchernen Korrekturen bis ins Adoleszentenalter abzuwarten und den betroffenen Kindern eine Kindheit mit schmerzhaft deformierten Füßen zuzumuten. 
Konservative Behandlungsmaßnahmen sind beim flexiblen Knick-Senk-Fuß nur in Ausnahmefällen angezeigt. Eine „reflektorische“ Verordnung von Einlagen oder Orthesen - seien sie propriozeptiv oder klassisch konstruiert - muss kritisch hinterfragt werden. Funktionseinschränkende hyperlaxe oder rigide Knick-Platt-Füße können dagegen durchaus - zumindest temporär - von einer adäquaten 3-dimensional wirkenden Orthesenversorgung profitieren. Allerdings sollte man sich von der Wirksamkeit der Maßnahme überzeugen und ihre Passform regelmäßig überprüfen.

Der idiopathische Knick-Platt-Fuß steht hinsichtlich der operativen Verfahren ganz im Zeichen der Arthrorise, einer wenig invasiven und dennoch effektiven Methode. Diese Tatsache birgt aber die nicht unerhebliche Gefahr in sich, dass eine größere Zahl asymptomatischer Füße mit guter Spontanprognose unkritisch operiert wird.

Wir würden die Operationsindikation für lockere KnickPlatt-Füße nur bei wirklich ausbleibender spontaner Verbesserungstendenz, bei entsprechenden Beschwerden und bei einseitigen Befunden stellen. Im Gegensatz dazu ist die Korrektur struktureller steifer Knick-Platt-Füße überaus anspruchsvoll und sollte frühzeitig durchgeführt werden.

Die operative Korrektur symptomatischer kindlicher Knick-Platt-Füße mit beginnender struktureller Fixierung (Rückfußequinus und -valgus, Vorfußsupinatus) ist aufwendig und muss meist als Kombinationseingriff geplant werden, da mehrere Ebenen der Deformität zu korrigieren sind. Die wichtige Kardanfunktion des Rückfußes darf bei neuromotorisch gesunden Kindern wenn möglich nicht angetastet, d.h. versteift werden. Der mittigen Verlaufsrichtung der Achillessehne kommt in dieser Hinsicht eine zentrale Bedeutung zu.

Sekundäre Knick-Platt-Füße erfordern ähnlich wie Klumpfüße meist eine frühzeitige kombinierte Formund Funktionskorrektur, wobei die Behandlung steifer oder instabiler Gelenkdeformitäten meist nur durch die Arthrodese möglich ist. Eine begleitende Spitzfußkomponente muss so schonend wie möglich im Interesse der Erhaltung einer ausreichenden Kraft der Wadenmuskulatur mitkorrigiert werden.

- Die erfolgreiche Behandlung einer kindlichen Fußdeformität stellt nicht den Endpunkt der Therapie dar. Sowohl das Wachstum als auch die primäre und oft weiter wirksame Pathologie machen entsprechende regelmäßige 6-12-monatige Verlaufskontrollen zur Pflicht.

\section{Komplikationen (Früh- und Spätkomplikationen)}

Sowohl bei der konservativen Therapie als auch durch operative Maßnahmen können Komplikationen auftreten, die sich jedoch in den meisten Fällen gut beherrschen lassen.

Die Überkorrektur von Klumpfüßen kann durch konservative wie auch operative Techniken hervorgerufen werden.

Ein in einen Hacken- oder Knickfuß überkorrigierter Klumpfuß stellt unseres Erachtens in jedem Falle eine zeitige Operationsindikation dar, deren Art und Dosierung sich nach den einzelnen Komponenten der neu entstandenen Deformität richten muss. Die präoperative Planung sollte - wenn möglich - immer auch dynamische Untersuchungen wie die Pedobarografie und die Ganganalyse beinhalten, da eine rein klinische und radiologische Beurteilung kaum alle Teilkomponenten der Deformität erfassen kann. Bei ausgeprägter Destabilisierung der Rückfußgelenke können stabilisierende Maßnahmen auch beim neuromotorisch gesunden Kind notwendig sein. Wir haben dies meist nach kompletter Dissoziation von Talus und Kalkaneus infolge einer kompletten Lösung der talokalkanearen Bandverbindungen beobachtet.

Die Subluxation oder gar Luxation der Peronäalsehnen stellt eine nach unserer Ansicht viel zu wenig beachtete Komplikation überkorrigierter Klumpfüße dar. Sie entsteht entweder durch die primär zu großzügige Lösung der peronäalen Retinacula hinter der Fibula oder sekundär als Folge einer Lateralabweichung des Kalkaneus. Die Peronäalsehnen werden auch beim Versuch der Kompensation einer zu schwachen Wadenmuskulatur als Hilfsmuskeln aktiviert. Ihre Subluxation oder gar Luxation führt zum Muskelungleichgewicht am ersten MTC-Gelenk durch die Insuffizienz der senkenden Wirkung des M. peronaeus longus mit der Folge eines Überwiegens des Tibialis anterior und einer Vorfußsupination. Zudem unterstützen nach ventral luxierte Peronäalsehnen die Hackenfußstellung als Fußheber (Abb.5).

Unterkorrekturen oder Klumpfußrezidive sind auch in Zeiten des Ponseti-Verfahrens nicht selten. Ihre Behandlung ist meist aufwendig und sollte wieder alle Komponenten (Rück-, Mittel- und Vorfuß) der jeweiligen Deformität umfassen und sorgfältig geplant werden. Die unzureichende Beachtung eines Vorfußspitzfußes, einer Vorfußpronation oder einer Vorfußsupination sind uns besonders häufig begegnet. 


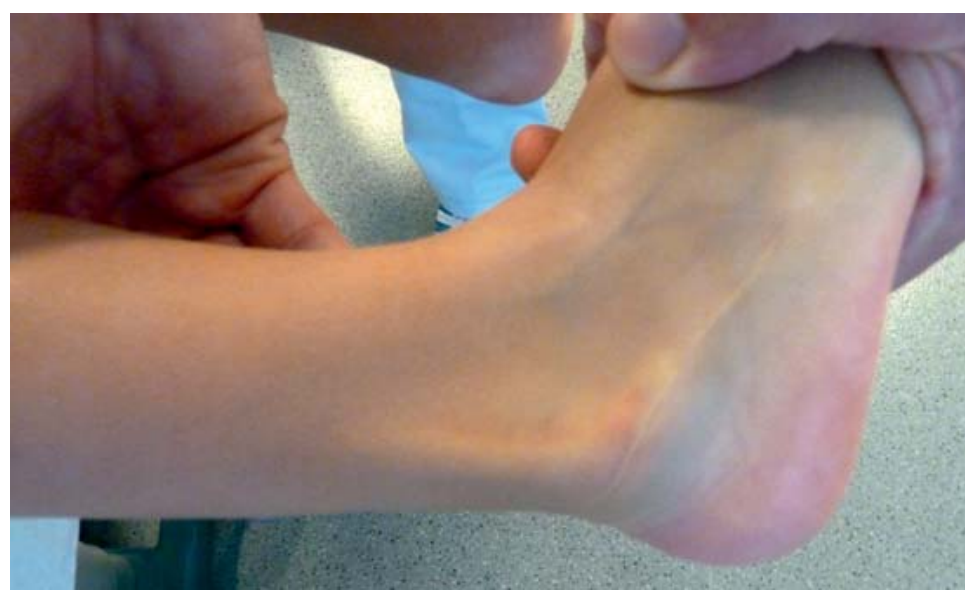

Abb. 5 - Peronealsehnenluxation.

Intra- und postoperative Komplikationen beinhalten bei perkutaner Ponseti-Technik oder bei der Korrektur von vernarbten Rezidivklumpfüßen die seltenen Gefäß- oder Nervenschädigungen, die umgehend revidiert werden sollten, und Wundheilungsstörungen durch eine zu starke Hautspannung medial oder dorsal. Hier sollte primär mit Kunsthaut gedeckt werden und der sekundäre plastische Hautverschluss angeschlossen werden.

Durch eine individuelle Planung der Operationsschritte, eine atraumatische Operationstechnik unter Verwendung von Haltefäden anstelle scharfer Haken, die Gelenkstabilisierung mit temporären Kirschner-Drähten und eine zeitlich befristete Anwendung der Blutsperre lassen sich die meisten Komplikationen vermeiden.

Komplikationen nach operativer Klumpfußkorrektur sind im Zeitalter der Ponseti-Technik selten geworden. Die früher weit verbreitete peritalare Arthrolyse hat uns dagegen eine Vielzahl unterschiedlichster Über- und Fehlkorrekturen beschert, die meist ebenfalls wieder operationspflichtig sind. Die wesentlichste Komplikation der operativen Knickfußtherapie besteht im Rezidiv bzw. in der Unterkorrektur.

\section{Nachbehandlung nach Operationen}

Die Dauer und die Art der Nachbehandlungen richten sich nach den Heilungseigenschaften der operierten Strukturen und der Gefahr von Überkorrekturen oder Rezidiven. Muskeln und Sehnen sind im Kindesalter nach spätestens 4-6 Wochen geheilt, Knochenoperationen nach 6 -10 Wochen, allerdings besteht besonders beim
Einfügen von Knochenkeilen noch eine längere Umbauphase, in der die operierten Strukturen geschützt werden müssen. Auch transponierte Sehnen können innerhalb der ersten Monate noch elongieren. Eine besondere Bedeutung kommt der frühzeitigen operativen Mobilisation eingesteifter bzw. bewegungseingeschränkter Gelenke zu. Hier muss zum Erreichen einer angestrebten Bewegungsverbesserung frühzeitig, $d$.h. bereits nach $1-2$ Wochen, aus gedeckelten Oberschenkelgipsen heraus mobilisiert werden, was nur durch die temporäre Stabilisierung der Rückfuß- und Chopart-Gelenke mit Kirschner-Drähten möglich ist. Nach Drahtentfernung (d.h. nach etwa 4-6 Wochen) muss die geführte Mobilisation über mehrere Monate kontinuierlich fortgesetzt werden, um die drohende erneute Vernarbungs- und Einsteifungsgefahr zu bannen. Dies kann nur über orthetisch unterstützte gezielte Mobilisationstechniken (Gelenkorthesen mit Quengelfedern oder einstellbaren Motoren) erfolgreich sein. Eine operative Gelenkmobilisation darf keinesfalls anschließend für 6 Wochen ununterbrochen ruhiggestellt werden, da damit das Risiko der erneuten Einsteifung

erheblich ist.

Die meisten operativen Fußkorrekturen im Kindesalter werden mit Gipsen vorübergehend immobilisiert und stabilisiert. Bei Säuglingen und bei allen ausgeprägten Klumpfußkorrekturen im Kleinkindesalter empfehlen wir unbedingt Oberschenkelgipse anzulegen, da nur damit eine adäquate Stellungskontrolle möglich ist. Alle Gelenkkorrekturen wie auch alle knöchernen Umstellungsoder Stabilisierungstechniken erfordern die temporäre Kirschner-Draht-Fixation der erreichten Korrekturstellung. Diese muss intraoperativ dokumentiert sein.

Die Gipsruhigstellung sollte in keinem Fall über die 6-Wochen-Grenze hinausgehen, da die negativen Effekte der länger dauernden Ruhigstellung auf Knochen, Gelenke und Muskulatur sonst nur schwer wieder rückgängig zu machen sind. Fixierende Kirschner-Drähte werden in Kurznarkose entfernt, nachdem die Nachbargelenke (meist OSG) zuvor noch einmal mobilisiert wurden. Anschließend sollte in derselben Kurznarkose der Abdruck für eine funktionelle Orthese mit Gelenken angefertigt werden.

Wir würden nach operativer Korrektur komplexer Fußdeformitäten stets für 9-12 Monate gelenktragende Orthesen in entsprechender 3-dimensional angepasster Korrekturstellung empfehlen, um die Gefahr des Korrekturverlusts bis zum Zeitpunkt der absolut stabilen knöchernen Konsolidierung und der muskulären Erholung gering zu halten. 
Auch nach scheinbar einfachen Operationen wie Muskelund Sehnenverlängerungen empfehlen wir wegen der schwächenden Wirkung dieser Verfahren und der Gefahr einer Elongation operierter Strukturen temporär Orthesen mit einer Freigabe erwünschter und einer Blockierung unerwünschter Bewegungsumfänge, um die Anpassung der Muskel-Sehnen-Einheit an die neue Gelenkstellung zu unterstützen und Überkorrekturen wie auch Rezidive zu vermeiden. Die orthetische Nachbehandlung wird durch die begleitende Physiotherapie zur Gelenkmobilisation und Muskelkräftigung wirkungsvoll unterstützt.

In den meisten Fällen operativer Fußkorrekturen können nach Ablauf eines Jahres wieder Kaufschuhe getragen werden, es sei denn, eine orthetische Behandlung ist wegen neurologischer Ausfälle weiterhin erforderlich.

- Die adäquate postoperative Therapie umfasst die Gipsbehandlung, die Orthetik und die krankengymnastische Mobilisation, die insbesondere bei Rezidivoperationen zum Einsatz kommt. Alle 3 Techniken sind komplex und machen eine spezielle Ausbildung notwendig, um ausreichend wirksam zu sein. Die gezielte Überprüfung der Wirksamkeit erspart dem Patienten wie dem Arzt unnötige Enttäuschungen.

\section{Prognose der verschiedenen Behandlungen}

Die Ergebnisse der klassischen ausgedehnt operativen Klumpfußbehandlungen sind insbesondere im Langzeitverlauf weniger günstig als die nach Ponseti-Therapie (van Gelder et al. 2010, Laaveg et al. 1980). Allerdings schwankte das operative Vorgehen überaus stark zwischen den klassischen posteromedialen und den peritalaren Techniken, was die Vergleichbarkeit erschwert.

Die Prognose korrekt durchgeführter operativer primärer Korrekturen ist bei entsprechend unterstützender Nachbehandlung und regelmäßigen Verlaufskontrollen in maximal 12-monatigen Abständen günstig.

Alle Operationen, die bei Rezidiven oder bei Überkorrekturen vorgenommen wurden, sind dagegen deutlich ungünstiger zu sehen und erfordern neben den meist erheblich umfangreicheren Eingriffen auch aufwendigere Nachbehandlungsmaßnahmen. Dennoch wird man das Ergebnis, das nach einer Primärkorrektur möglich gewesen wäre, meist nicht mehr erreichen. Der Grund hierfür sind Vernarbungen, Muskelschwäche und Versteifungen durch den Primäreingriff.
Je geringgradiger die primäre Deformität, umso günstiger die Prognose. Der primäre lockere Knick-Platt-Fuß hat eine überwiegend günstige Prognose, ob er nur beobachtet oder konservativ bzw. operativ behandelt wird. Auch der primäre Klumpfuß kann mit der korrekten Anwendung der Ponseti-Technik in über 90\% der Fälle dauerhaft korrigiert werden, allerdings bleiben stets die Wadenmuskelschwäche und eine leichte Bein- und Fußverkürzung zurück.

Strukturelle Klumpfüße beim älteren Kind haben hinsichtlich der Stellungskorrektur eine relativ günstige Prognose, die Beweglichkeit wird jedoch ebenso wie die Muskelkraft dauerhaft reduziert bleiben.

Bei allen sekundären Fußdeformitäten richtet sich die Prognose nach der Grunderkrankung (stationär oder progredient) und nach der Art und dem Ausmaß der Deformität und der operativen Therapie. Wenn neben der pathologischen Stellung auch das gestörte Muskelgleichgewicht berücksichtigt werden konnte und die Gelenke in stabiler Mittelstellung verbleiben, so ist die Prognose auch längerfristig günstig. Allerdings werden sowohl bei primären als auch besonders bei sekundären Fußdeformitäten immer regelmäßige Nachkontrollen wenigstens in jährlichen Abständen während des Wachstums notwendig sein, um eventuelle Verschlechterungen oder Überkorrekturen rechtzeitig erkennen und ggf. behandeln zu können.

Die Prognose konservativer Verfahren ist nur bei geringfügigen Deformitäten günstig, wenn die natürliche Entwicklung der Deformität die Behandlung günstig begleitet.

Kurzfristige Behandlungsergebnisse sind in der Kinderorthopädie fast immer nur von geringem Wert. Nur die regelmäßige Überprüfung der Resultate nach Form und Funktionsergebnis vermag ein klares Bild über die Behandlungsqualität zu liefern. Das Wachstum kann insbesondere in den Phasen der raschen Größen- und Gewichtsprogression so manches schöne Resultat zunichte machen. Immer dann sind die erneute Indikationsstellung und adäquate Therapieauswahl besonders wichtig.

\section{Begutachtung/gutachterliche Aspekte}

Im Kindesalter sind gutachterliche Aspekte hinsichtlich der Behandlung von Fußdeformitäten selten. Es muss aber stets daran gedacht werden, dass die Voraussetzungen für eine normale Fußform und -funktion bei allen strukturellen Deformitäten fehlen. Deshalb muss immer 
schon vor dem Einleiten einer Therapie ein realistisches kurz-, mittel- und falls möglich auch längerfristiges Therapieziel definiert werden, ehe die dazu notwendigen Maßnahmen ausgewählt und angewendet werden. Je schwerer die ursprüngliche Fußdeformität war und je später mit der Behandlung begonnen wurde, aber auch wenn Voroperationen erfolglos verlaufen sind, umso bescheidener wird das erreichbare Behandlungsziel neu zu definieren sein.

Als minimales Therapieziel wird man aber für die meisten Fußdeformitäten eine plantigrade Fußstellung, einen korrekt ausgerichteten (d.h. symmetrischen) Fußöffnungswinkel, keine plantaren Druckstellen, keine Schmerzen und, falls möglich, auch eine ausreichende Beweglichkeit der funktionell wichtigen Gelenke (OSG, Zehengrundgelenke) definieren können.

Ob Kaufschuhe möglich sind oder Orthesen getragen werden müssen, hängt auch vom Zustand der proximalen Gelenke bzw. der Gegenseite ab und insbesondere, ob gleichzeitig auch neurologische Störungen vorliegen.

\section{Zukunftsperspektiven für Diagnostik und Therapie}

Die Ausblicke auf Zukunftsentwicklungen bei der Behandlung kindlicher Fußdeformitäten sind vielschichtig. Durch moderne bildgebende Verfahren gelingt die frühzeitige sonografische oder MRT-technische Formdiagnose von Fußdeformitäten bereits im Mutterleib. Die Art und der Umfang der einzuleitenden Therapien werden jedoch immer erst nach der Geburt anhand der klinischen Untersuchungen festgelegt werden können. Eine Standardisierung von Therapien, wie sie bei der Ponseti-Methode bereits etabliert ist, wäre auch für andere Deformitäten wünschenswert. Allerdings nimmt der angeborene Klumpfuß hinsichtlich seiner Häufigkeit und Ausprägungsform wie auch seiner Behandlungsbedürftigkeit eine Sonderstellung ein. Der idiopathische Knick-PlattFuß kann heute noch nicht anhand sicherer Prädiktoren in gutartig oder behandlungsbedürftig eingeteilt werden, was den Prozentsatz überflüssiger Behandlungen sicher erhöht.

Hier sind Longitudinalstudien ebenso nötig wie für die Bewertung des längerfristigen Effekts konservativer Therapieverfahren.
Für die Indikationsstellung zu operativen Techniken und die funktionelle Evaluation scheinen sich dynamische Messverfahren der Fußfunktion zunehmend durchzusetzen. Allerdings ist der technische und personelle Aufwand dazu nicht unerheblich, ganz abgesehen von der Pflicht für den Operateur, sich auf diesem Spezialgebiet ständig weiterzubilden.

Die Verwendung von Scores zur Verlaufskontrolle bleibt trotz vielfältiger Einschränkungen bis auf Weiteres noch Standard. Aber auch dafür ist der Aufwand nicht gering einzuschätzen.

Hinsichtlich der Entwicklung neuer Operationsverfahren bleibt wohl weniger zu erwarten, sieht man von neuen Osteosynthesematerialien einmal ab. Hier ist es eher die gezielte Kombination etablierter Verfahren zur Korrektur kombinierter Deformitäten und deren korrekte Anwendung, die neu zu definieren sein wird.

Schließlich lassen neue dynamische Gelenkorthesen und der Einbau von programmierbaren Motoren bzw. Rückstellkräften weitere Verbesserungen, besonders für die postoperative Versorgung von komplexeren Deformitäten, erwarten. 


\section{Quellenangaben}

Dobbs MB, Purcell DB, Nunley R et al. Early results of a new method of treatment for idiopathic congenital vertical talus. Surgical technique. J Bone Joint Surg Am 2007; 89: 111 - 121

Laaveg SJ, Ponseti IV. Long term results of treatment of congenital dubfoot. J Bone Joint Surg 1980; 62A: 23-30

van Gelder JH, van Ruiten AGP, Viser JD et al. Long-term results of the posteromedial release in the treatment of idiopathic clubfoot. J Pediatr Orthop 2010; 30: 700-704

\section{Weiterführende Literatur}

\section{Klumpfuß}

Boehm S, Limpaphayom N, Alaee F et al. Early results of the Ponseti method for the treatment of clubfeet in distal arthrogryposis. J Bone Joint Surg 2008; 90 A: 1501 - 1507

Brückner U. Klumpfüßchen-Therapie nach Ponseti. 2. Aufl. Aachen: Shaker Verlag; 2010

Chotel F, Parot R, Seringe R et al. Comparative study: Ponseti method versus french physiotherapy for initial treatment of idiopathic clubfoot deformity. J Pediat Orthop 2011; 31: 320-325

Döderlein L, Wenz W, Schneider U. Der Klumpfuß. Berlin: Springer; 1999 Exner GU, Anderhuber F, Haldi-Brändle V et al. Klumpfuß-Pathoanatomie, manuell-funktionelle und operative Behandlung. Darmstadt: Steinkopff; 2005

Jowett CR, Morcuende JA, Ramachandran M. Management of congenital talipes equinovarus using the Ponseti method. J Bone Joint Surg 2011; 93 B: $1160-1164$

Laaveg SJ, Ponseti IV. Long term results of treatment of congenital dubfoot. J Bone Joint Surg 1980; 62A: 23-30

Mehrafshan M, Rampal V, Seringe R et al. Recurrent club foot deformity following previous soft tissue release. J Bone Joint Surg 2009; 91 B: 949-955

Ponseti IV. Congenital clubfoot. Oxford: Oxford University Press; 1996

Richards BS, Faulks S, Rathien KE et al. A comparison of two nonoperative methods of idiopathic clubfoot correction: the Ponseti method and the French functional method. J Bone joint Surg 2008; 90 A: 2313-2321

van Gelder JH, van Ruiten AGP, Viser JD et al. Long-term results of the posteromedial release in the treatment of idiopathic clubfoot. J Pediatr Orthop 2010; 30: 700-704

Weimann-Stahlschmidt K, Krauspe R, Westhoff B. Kongenitaler Klumpfuß. Orthopäde 2010; 39: 1071-1086

\section{Knick-Platt-Fuß}

Dobbs MB, Purcell DB, Nunley R et al. Early results of a new method of treatment for idiopathic congenital vertical talus. Surgical technique. J Bone Joint Surg Am 2007; 89: 111-121

Döderlein L, Wenz W, Schneider U. Der Knickplattfuß. Berlin: Springer; 2002

Hamel J. Radiologisch-dokumentierte Korrektureffekte beim kindlichen Pes planovalgus mit der Calcaneo-Stop-Arthrorise und der Calcaneus-Verlängerungsosteotomie. Fuß \& Sprunggelenk 2010; 8: 43-47

Moraleda L, Mubarak SJ. Flexible flatfoot: Differences in the relative alignment of each segment of the foot between symptomatic and asymptomatic patients. J Pediatr Orthop 2011; 31: 421 - 428

Staheli LT, Chew DE, Corbett M. The longitudinal arch: a survey of 882 feet in normal children and adults. J Bone Joint Surg 1987; 69 A: $426-428$

Vanderwilde R, Staheli LT, Chew DE et al. Measurements on radiographs of the foot in normal infants and children. J Bone Joint Surg 1988; 70 A: $407-415$

Wenger DR, Mauldin D, Speck G. Corrective shoes and inserts as treatment for flexible flatfoot in infants and children. J Bone Joint Surg 1989; 71 A: $800-810$

Westhoff B, Weimann-Stahlschmidt K, Krauspe R. Der Knicksenkfuß im Kindesalter: Pathomorphologie, Spontanverlauf, konservative Behandlungsansätze. Fuß \& Sprunggelenk 2010; 8: 5-15

\section{Korrespondenzadresse}

\section{Dr. L. Döderlein}

Orthopädische Kinderklinik

am Behandlungszentrum Aschau $\mathrm{GmbH}$

Bernauerstr. 18

83229 Aschau im Chiemgau

Telefon: 08052/171-2000

Fax: $\quad$ 08052/171-2099

E-Mail: I.doederlein@bz-aschau.de 


\section{Pädiatrische Orthopädie und Unfallchirurgie}

\section{CME-Fragen}

Welche Aussage ist richtig?

Es besteht keine typische Indikation zur Fußkorrektur bei ...

\section{CME•thieme.de}

\section{CME-Teilnahme}

- Viel Erfolg bei Ihrer CME-Teilnahme unter http://cme.thieme.de

- Diese Fortbildungseinheit ist 12 Monate online für eine CME-Teilnahme verfügbar.

- Sollten Sie Fragen zur Online-Teilnahme haben, unter http://cme.thieme.de/hilfe finden Sie eine ausführliche Anleitung.

\section{Welche Aussage trifft zu?}

Es bestehen folgende Kontraindikationen für eine operative Fußkorrektur:

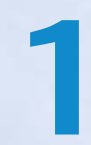

A Funktionseinschränkung durch die Deformität.

B Schmerzen.

C erfolgloser konservativer Behandlung.

D kosmetischer Beeinträchtigung.

E Coalitio talocalcanearis.
Folgende Aussage ist falsch:

Welche Aussage zur Klumpfußbehandlung ist falsch?
A Rezidivfehlstellung.

B primär nicht auskorrigierter Fuß.

C Synostosen.

D lähmungsbedingte flexible Fehlstellung ohne transferfähige Muskulatur beim immobilen Patienten.

E Muskelungleichgewicht.

D Nach abgeschlossener Redressionsbehandlung muss bis zum 4. Lebensjahr eine Orthesenbehandlung erfolgen.

E Klumpfüße sind bis zum Wachstumsabschluss zu kontrollieren. 
A Ein einseitiger Knick-Senk-Fuß ist unbedenklich.

B Auch bei Vorliegen einer Coalitio ist eine konservative Therapie sinnvoll.

C Der flexible Knick-Senk-Fuß im Kindesalter ist in der Regel nicht behandlungsbedürftig.

D Nur selten besteht beim Knick-Senk-Fuß eine Hyperlaxizität.

E Der Knick-Senk-Fuß ist beim Kleinkind und Jugendlichen meist schmerzhaft.

Welche Aussage zur peritalaren Arthrolyse beim Klumpfuß trifft nicht zu:
A Die peritalare Arthrolyse stellt die klassische Klumpfußoperation dar.

B Ziel ist, die strukturelle Verwringung der subtalaren Fußplatte wieder zu normalisieren.

C Eine Kirschner-Draht-Fixation ist nicht erforderlich.

D Achillessehne und M.-Tib.-post.-Sehne werden dosiert verlängert.

E Ein Oberschenkelgips wird postoperativ für die Dauer von 6 Wochen erforderlich.
Welche Aussage trifft zu?

Folgende Aussagen zur operativen Fußkorrektur treffen zu?
A Bei der Korrektur von Knick-Platt-Füßen muss die peritalare Entwringung verstärkt werden.

B Bei der OP nach Evans wird die laterale Fußsäule verkürzt.

C Eine vorhandene Vorfußadduktion wird durch die OP nach Evans verbessert.

D Bei der operativen Knick-Platt-Fuß-Korrektur ist häufig auch eine Fersenbeinverschiebung nach medial indiziert.

E Eine eventuell vorhandene Vorfußsupination spielt für die Korrektur keine Rolle. 


\section{Pädiatrische Orthopädie und Unfallchirurgie}

Folgende Aussage zur postoperativen Nachbehandlung ist falsch?
A Muskeln und Sehnen sind nach 4-6 Wochen geheilt.

B Knöcherne Korrekturen sind nach 6-10 Wochen geheilt.

C Nach Primäreingriffen wird keine Orthesenversorgung erforderlich.

D Versetzte Sehnen müssen durch Orthesen geschützt werden.

E Nach Rezidiveingriffen besteht eine erhöhte Vernarbungsgefahr.
Welche Aussage ist falsch? Die OP-Aufklärung sollte folgende Punkte umfassen:

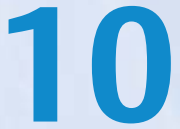

A allgemeine OP-Risiken.

B deformitätsspezifische Risiken einschließlich Rezidiv, Über- und Unterkorrektur.

C Darstellung der Korrekturprinzipien mit möglichst einfachen und verständlichen Worten, ggf. OP-Skizze.

D Die Patienten bzw. Eltern dürfen nicht unter Zeitdruck gesetzt werden, da es sich in aller Regel um Wahleingriffe handelt.

E Eine ausführliche Aufklärung ist nicht sinnvoll, da die Operationsprinzipien für medizinische Laien zu komplex sind. 